(2) Open Access Full Text Article

\title{
Impact of the COVID-19 Pandemic on Family
} Wellbeing in the Context of Neurodevelopmental Disorders

\author{
Renata Rizzo' \\ Lisa Karlov 2,3 \\ Nicoletta Maugeri ${ }^{1}$ \\ Selena di silvestre \\ Valsamma Eapen (iD) 2,3 \\ 'Child and Adolescent Neurology and \\ Psychiatry, Department of Clinical and \\ Experimental Medicine, Catania \\ University, Catania, Italy; ${ }^{2}$ School of \\ Psychiatry, UNSW, Sydney, NSW, \\ Australia; ${ }^{3}$ Academic Unit of Child \\ Psychiatry South West Sydney, Liverpool \\ Hospital \& Ingham Institute, Sydney, Nsw, \\ Australia
}

Correspondence: Valsamma Eapen Email v.eapen@unsw.edu.au
Objective: The primary aim was to assess both the type and degree of impact of the COVID-19 pandemic on child and family wellbeing in a cohort with neurodevelopmental disorders.

Design: This was a single time-point observational study utilizing a combination of surveys and standardized measures, which were administered to parents by researchers by telephone. Setting: The Child and Adolescent Neuropsychiatric Clinic of the Department of Clinical and Experimental Medicine, Catania University, Italy.

Participants: In total, 200 caregivers completed the questionnaires on behalf of themselves and their child. They were predominantly mothers $(88.00 \%)$ and primary caregivers $(93.50 \%)$, with a mean age of 42.84 years $(\mathrm{sd}=7.13)$.

Primary and Secondary Outcomes Measures: A questionnaire featured in a previous study was used to assess the impact of COVID-19 on general wellbeing, types of support, family health, home-based learning, and child behaviors. Children's diagnoses were recorded. Caregivers provided information about physical, mental, financial, and vocational wellbeing, and completed several standardised measures of mental health and well-being: the Kessler Psychological Distress Scale - K6; the General Anxiety Disorder Scale - GAD-2; and the WHO Well-being Scale - WHO-5.

Results: Overall, $58.50 \%$ of respondents agreed somewhat or strongly that their child's overall health and wellbeing had been impacted by the pandemic, while $47.74 \%$ felt that their own wellbeing as parents had been affected. Whilst home-based learning and disruption to services for children were noted as being significant, child wellbeing appeared to not be correlated with these but rather to restrictions, home isolation, and disruption to routine.

Conclusion: Children with neurocognitive disorders and their families have been substantially impacted by the COVID-19 pandemic. It is expected that targeted resources and support services will be required in response to this increase in need.

Keywords: neurocognitive, K6, GAD-2, WHO-5, general wellbeing

\section{Introduction}

The global pandemic caused by COVID-19 has created rapid changes to how people are able to carry out their normal lives, with impacts ranging from health and mortality through to those impacts brought about by social isolation rules and localized lockdowns. Concern has been expressed over the impact of lockdown and isolation in particular on child and adolescent mental health. ${ }^{1}$ Whilst research on this topic has been sparse prior to the current time of pandemic, a number of previous studies highlighted in a recent review have established that there are 
changes in children's access to play during times of crisis and isolation, and that this in turn can impact a child's well-being. ${ }^{2}$

Early results from research studies focusing on the observed impact of COVID-19 have indicated that adolescents may show an increase in symptoms of depression and anxiety, and that this increase is tied to restrictions designed to contain the spread of the pandemic. ${ }^{3}$ A study conducted in the United States found that around a third of school-aged children had demonstrated problematic behavior changes since the start of the pandemic and that parents were also reporting symptoms in line with depression, anxiety, and stress, which in turn predicted poorer child outcomes. ${ }^{4}$ Meanwhile, a Canadian study found two thirds of school-aged children experiencing deteriorating mental health, with higher rates for children with preexisting mental health diagnoses, associated in particular with stress due to social isolation. ${ }^{5}$ It appears from these initial results that disruption to the established behaviors of children and adolescents around the world is having a deleterious effect on their mental health.

Parents, too, appear to be impacted by the society-wide secondary effects of COVID-19. A large European study reported that parents experienced deteriorating wellbeing associated with homeschooling, which appeared to be largely consistent across the seven countries included and across families with or without a child diagnosed with a mental health condition. ${ }^{6}$ With respect to prior instances of pandemics, a systematic review found that increased age of parents and higher socio-economic scores were protective factors against a mental health impact of a pandemic, though isolation and quarantine were both highlighted as common causes of increased mental health problems amongst children. ${ }^{7}$ Similarly, an Italian study found that older adults and those with the capacity to adapt did not experience an increase in psychopathology during lockdown, but that females had a slightly higher incidence of increasing symptoms of depression, anxiety, and circadian rhythm disruption than males. ${ }^{8}$ These results together appear to suggest that mothers who are younger and of lower socio-economic status may be at particular risk for impacts to mental health and wellbeing during COVID-19.

However, for some groups of children and parents, there is a pre-existing burden of care and treatment need. It is thought probable that families with a background of caring for a child with a neurodevelopmental or psychological disorder may experience increased adverse effects from society-wide disasters such as a pandemic. Indeed, it has been reported that families of children with developmental disabilities have experienced additional challenges given that their children can find rules around mask wearing and social distancing to be particularly difficult, and given that disruption in service provision to children can be particularly impactful for a family. ${ }^{9}$ Another study focusing on parents of children with neurodevelopmental disorders, found that concern for children having to go without their usual treatment and support programs during the pandemic was related to poorer parental mental health. ${ }^{10}$

The above themes of child and parental mental health and wellbeing in families caring for a child with a neurodevelopmental or psychological disorder were explored in an Australian sample earlier in the pandemic, where it was found that $76.9 \%$ of families experienced an impact on some aspect of their child's health and wellbeing. ${ }^{11}$ We take up the same questions in the current study as we turn the lens on a sample of parents of children in Italy with primarily neurodevelopmental disorders. In particular, this study aims to explore whether parents see a change in their own mental health and wellbeing and in their child's health and wellbeing during the COVID-19 pandemic, whilst considering the specific changes to routine, services, lifestyle, and other factors that families may experience.

\section{Methods}

A cross-sectional quantitative study (survey) was used at one timepoint. Parents and caregivers of a child with a neurodevelopmental condition (mainly Tourette Syndrome and Autism Spectrum Disorder) were invited to participate in the survey through the Child and Adolescent Neuropsychiatric Clinic of the Department of Clinical and Experimental Medicine, Catania University. Parents/ caregivers completed the survey questionnaire, similar to that used in an Australian population, ${ }^{1}$ via telephone. The study received approval from the Catania University Ethics Committee and was conducted in accordance with the Declaration of Helsinki. Parents gave their informed consent.

\section{Child Symptom Severity and Well-Being}

Respondents were asked to report all relevant diagnoses their child had received, together with a rating of symptom change since the start of the pandemic. A five-point scale was used: 1 "symptoms much improved", 2 "symptoms 
somewhat improved", 3 "symptoms the same", 4 "symptoms somewhat worse", 5 "symptoms much worse". As with the previous study utilizing this questionnaire, we combined responses of "somewhat worse" and "much worse" into one group indicating worsening of the child's situation. Further questions were included relating to: (i) general wellbeing (perceived impact of the pandemic across the family); (ii) types of support (including professional assistance and social connection); (iii) family health (employment, finances, food, and housing); (iv) home based learning (challenges in continuing education while a child is remaining in the family home); and (v) child behaviors (including emotional responses and use of technology/devices). All of the above questions used a fivepoint response scale ranging from "strongly agree" to "strongly disagree". Again, the two responses at the impacted end of the scale - "strongly agree" and "somewhat agree" - were considered together as representing the larger group of respondents feeling a negative impact.

Parents/Caregivers were also asked about their own physical and mental health and wellbeing, any impact on their employment, stability of accommodation and any financial impact as well as their experience of work-life balance during the pandemic. In addition, they completed the following standardised measures of mental health and well-being: the 6 item Kessler Psychological Distress Scale - K6; ${ }^{12}$ the 2 item General Anxiety Disorder Scale - GAD-2, ${ }^{13}$ the 5 item WHO Well-being Scale WHO-5. ${ }^{14}$

\section{Results}

A total of 200 caregivers completed the questionnaire. They were predominantly mothers $(88.00 \%)$ and primary caregivers $(93.50 \%)$, with a mean age of 42.84 years $(\mathrm{sd}=$ $7.13)$. The questionnaire respondents were educated, with $70.00 \%$ having completed further education after finishing high school. The majority (42.50\%) identified home duties as their primary occupation, whilst $30.50 \%$ were currently working for employers and $18.00 \%$ were on temporary leave due to COVID-19. Those with partners (90.50\%) generally reported that their partner was either currently employed $(58.00 \%)$ or on temporary leave due to COVID19 (21.50\%). A full $97.99 \%$ reported being under social isolation at the time of responding. A total of $16.50 \%$ of households reported one adult at least who was an essential worker; $2.50 \%$ reported having a frontline health care worker in their household. Only one family $(0.50 \%)$
Table I Diagnoses and Perceived Effect of Pandemic on Symptoms

\begin{tabular}{|l|r|r|r|}
\hline & $\mathbf{n}$ & $\%$ & $\begin{array}{r}\% \text { with } \\
\text { Worsening } \\
\text { Symptoms }\end{array}$ \\
\hline ADHD & 6 & 3.00 & 50.00 \\
Anxiety Disorder & 4 & 2.00 & 50.00 \\
Autism Spectrum Disorder & 102 & 51.00 & 37.25 \\
Genetic Disorder & 4 & 2.00 & 0.00 \\
Intellectual or Learning Disorder & 75 & 37.50 & 42.67 \\
Obsessive Compulsive Disorder & 35 & 17.50 & 54.29 \\
Tourette Syndrome & 109 & 54.50 & 52.29 \\
\hline
\end{tabular}

reported being split up from one another physically due to the pandemic.

The majority of the children were male $(81.50 \%)$ and ranged in age between 3 and 18 years old (mean $=10.74$ years, $s d=3.96$ ). The overwhelming majority were born in Italy $(99.00 \%)$ and $77.00 \%$ had siblings. As shown in Table $1,54.50 \%$ of the sample were diagnosed with Tourette's Syndrome, $51.00 \%$ held a diagnosis of Autism, and $37.50 \%$ were diagnosed with an intellectual or learning disorder. Particular impact on worsening of symptoms was perceived for the children with Obsessive Compulsive Disorder $(54.29 \%$ appeared to become worse), Tourette Syndrome (52.29\%), ADHD (50.00\%), and Anxiety Disorder (50.00\%).

Table 2 contains information regarding caregivers' selfreported wellbeing over the past 30 days prior to completing the questionnaire. As can be seen, $36.00 \%$ of those answering the question reported having been nervous most or all of the time, $25.00 \%$ reported feeling hopeless most or all of the time, and $30.50 \%$ reported feeling fidgety most or all of the time. A full $26.00 \%$ reported that most or all of the time they felt so depressed nothing could cheer them up, whilst $20.00 \%$ felt everything was an effort most or all of the time, and $15.00 \%$ felt worthless most or all of the time. With respect to the two weeks prior to responding, $19.00 \%$ had felt bothered by feeling anxious more than half of the days or more often, and $10.50 \%$ had been bothered by not being able to stop worrying. Only $9.00 \%$ reported having felt cheerful only some of the time or at no time, while $19.00 \%$ felt calm and relaxed either some of the time or at no time. A total of $6.50 \%$ felt active and vigorous some of the time or at no time, $15.50 \%$ reported waking up feeling fresh and rested only some of the time or at no time, and $20.00 \%$ felt their life had been 
Table 2 Caregivers' Mental Health and Wellbeing

\begin{tabular}{|c|c|c|}
\hline & $\mathbf{n}$ & $\%$ \\
\hline Kessler & & \\
\hline $\begin{array}{l}\text { During the past } 30 \text { days, how often did you feel } \\
\text { nervous? }\end{array}$ & 72 & 36.00 \\
\hline $\begin{array}{l}\text { During the past } 30 \text { days, how often did you feel } \\
\text { hopeless? }\end{array}$ & 50 & 25.00 \\
\hline $\begin{array}{l}\text { During the past } 30 \text { days, how often did you feel restless } \\
\text { or fidgety? }\end{array}$ & 61 & 30.50 \\
\hline $\begin{array}{l}\text { During the past } 30 \text { days, how often did you feel so } \\
\text { depressed that nothing could cheer you up? }\end{array}$ & 52 & 26.00 \\
\hline $\begin{array}{l}\text { During the past } 30 \text { days, how often did you feel that } \\
\text { everything was an effort? }\end{array}$ & 40 & 20.00 \\
\hline $\begin{array}{l}\text { During the past } 30 \text { days, how often did you feel } \\
\text { worthless? }\end{array}$ & 30 & 15.00 \\
\hline Generalized Anxiety Scale & & \\
\hline $\begin{array}{l}\text { Over the past two weeks, how often have you been } \\
\text { bothered by feeling nervous, anxious or on edge? }\end{array}$ & 38 & 19.00 \\
\hline $\begin{array}{l}\text { Over the past two weeks, how often have you been } \\
\text { bothered by not being able to stop or control } \\
\text { worrying? }\end{array}$ & 21 & 10.50 \\
\hline WHO (Five) Well-Being Index & & \\
\hline I have felt cheerful and in good spirits & 18 & 9.00 \\
\hline I have felt calm and relaxed & 38 & 19.00 \\
\hline I have felt active and vigorous & 13 & 6.50 \\
\hline I woke up feeling fresh and rested & 31 & 15.50 \\
\hline My daily life has been filled with things that interest me & 40 & 20.00 \\
\hline
\end{tabular}

filled with things that interested them only some of the time or at no time.

A number of questions were asked in relation to perceptions about how children have been affected by the pandemic; see Table 3. A full $58.50 \%$ of respondents agreed somewhat or strongly that their child's overall health and wellbeing had been impacted by the pandemic. Close to half $(45.50 \%)$ believed the pandemic had worsened preexisting health conditions for their child, while $36.36 \%$ felt the pandemic had an impact on their other children's wellbeing. A full 47.74\% felt either somewhat or strongly that their own wellbeing as parents had been affected.

With respect to support networks, 53.01\% felt their support networks had decreased and $44.89 \%$ felt that carer supports and services had decreased. Disruption in respite services had been felt by $27.08 \%$ of respondents, whilst $74.29 \%$ of those with children accessing allied health services had felt this access had been significantly disrupted. For families accessing psychological therapy, $56.00 \%$ had felt a disruption to access, whilst $70.73 \%$ of those accessing behavioral services felt access had been disrupted. The majority of carers did not report a change to medication related to the pandemic; roughly $25.24 \%$ felt their child had been taking more medication than usual, whilst 3.06\% felt their child had been taking less medication than usual. For children seeing specialists, $29.21 \%$ of families felt access had been disrupted, whilst $64.52 \%$ felt access to allied health services had been disrupted. Carers' ability to organize supports had been decreased for $28.08 \%$ of families, and children's ability to see people outside of the home had been reduced for $62.25 \%$.

With respect to the health of the family as a whole, $94.50 \%$ of respondents were hopeful that the pandemic would end soon. However, significant worries were reported about making it through future periods of isolation $(61.93 \%)$, about the stability of living situations $(26.26 \%)$, about running out of food due to lack of money (10.61\%), and about COVID-19 itself (61.62\%). Close to half $(46.97 \%)$ felt restrictions had been stressful for their child, whilst $60.10 \%$ had found home isolation to be personally stressful. A full $69.00 \%$ reported disruption to their child's routines, however only $7.07 \%$ reported a reduction in quality time spent with their child. A full $40.00 \%$ of carers reported difficulty in balancing all of their caring and working responsibilities, with $32.48 \%$ reporting worsening of mental health conditions and $28.57 \%$ reporting worsening of physical health conditions. Changes in employment status were reported by $48.48 \%$ and increased financial problems were reported by $32.83 \%$, with $57.58 \%$ reporting that COVID restrictions had a significant negative effect on their lives.

Home based learning was carried out by a reported $87.18 \%$ of children, with $90.26 \%$ prevented from attending school and $68.88 \%$ experiencing disruption to learning. However, whilst $84.02 \%$ of respondents believed they were equipped with technology to manage online learning, and $79.90 \%$ believed they were equipped to support their child's learning, only $47.45 \%$ felt their child was capable of engaging in home-based learning.

In the area of children's physical wellbeing, $18.69 \%$ of families reported a reduction in sleep quality, $67.50 \%$ reported a reduction in child's exercise, and $11.22 \%$ reported their child consuming a poorer diet. The majority of families $(80.90 \%)$ believed the pandemic had reduced their child's ability to spend time outside. Almost half (43.23\%) found it hard to maintain relationships during COVID, whilst $27.51 \%$ of children struggled to follow recommendations about social distance. Around a quarter of families 
Table 3 Impacts on Children and Families (Those Who Agree or Strongly Agree with Each Statement)

\begin{tabular}{|c|c|c|}
\hline & $\mathbf{n}$ & $\%$ \\
\hline \multicolumn{3}{|l|}{ General } \\
\hline My child's overall health and wellbeing has been impacted by the COVID-I9 crisis & 117 & 58.50 \\
\hline COVID has worsened pre-existing health conditions for my child & 91 & 45.50 \\
\hline COVID has had an impact on my wellbeing & 95 & 47.74 \\
\hline COVID has had an impact on my other children's wellbeing & 56 & 36.36 \\
\hline \multicolumn{3}{|l|}{ Types of support } \\
\hline My support networks have decreased significantly due to COVID & 97 & 53.01 \\
\hline COVID has disrupted carer supports and services & 79 & 44.89 \\
\hline COVID has disrupted respite arrangements & 52 & 27.08 \\
\hline $\begin{array}{l}\text { COVID has significantly disrupted the allied health services my child accesses (eg occupational therapy, speech } \\
\text { therapy) }\end{array}$ & 78 & 74.29 \\
\hline COVID has significantly disrupted my child's psychological therapy & 42 & 56.00 \\
\hline COVID has significantly disrupted my child's behavioural therapy & 58 & 70.73 \\
\hline My child has started taking more medication than normal due to the COVID pandemic & 26 & 25.24 \\
\hline My child is taking less medication than usual because of the COVID pandemic & 3 & 3.06 \\
\hline My child's ability to access specialists has been significantly impacted due to COVID & 52 & 29.21 \\
\hline My ability to organize supports has been significantly impacted due to COVID (eg support workers) & 41 & 28.08 \\
\hline COVID has significantly disrupted my child's other treatments and supports (eg physiotherapy, OT, speech therapy) & 60 & 64.52 \\
\hline My child's contacts with people outside of the home have reduced since before the COVID pandemic & 117 & 62.23 \\
\hline \multicolumn{3}{|l|}{ Family health } \\
\hline COVID has significantly disrupted my child's routines & 138 & 69.00 \\
\hline During the past two weeks, COVID restrictions have been stressful for my child & 93 & 46.97 \\
\hline Home isolation has been stressful for me & 119 & 60.10 \\
\hline Balancing work with childcare/family responsibilities has been difficult for me due to COVID & 74 & 40.00 \\
\hline My pre-existing mental health conditions have been worsened due to COVID & 38 & 32.48 \\
\hline My pre-existing physical health conditions have been worsened due to COVID & 34 & 28.57 \\
\hline My family is feeling worried about COVID & 122 & 61.62 \\
\hline The COVID restrictions have had significant negative effect on my life & 114 & 57.58 \\
\hline The amount of quality time I spend with my child has reduced since before COVID-19 & 14 & 7.07 \\
\hline Changes related to the Coronavirus/COVID-19 crisis have created financial problems for me or my family & 65 & 32.83 \\
\hline The COVID situation has resulted in changes to the employment status of people in my household & 96 & 48.48 \\
\hline I am concerned about the stability of our living situation & 52 & 26.26 \\
\hline I am worried about running out of food due to a lack of money & 21 & 10.61 \\
\hline I am hopeful that the COVID crisis will end soon & 189 & 94.50 \\
\hline I am worried about making it through future periods of isolation & 122 & 61.93 \\
\hline \multicolumn{3}{|l|}{ Home based learning } \\
\hline COVID has prevented my child from attending educational facilities in person (eg childcare, school) & 176 & 90.26 \\
\hline COVID has disrupted my child's learning & 135 & 68.88 \\
\hline My child has completed home-based learning during the COVID-19 pandemic. & 170 & 87.18 \\
\hline I have adequate access to internet and online resources & 163 & 84.02 \\
\hline My child has adequate capacity to engage in home based learning & 93 & 47.45 \\
\hline I have adequate capacity to support my child's educational needs & 155 & 79.90 \\
\hline \multicolumn{3}{|l|}{ Child behaviours } \\
\hline My child has experienced reductions in sleep quality as a result of COVID-19 & 37 & 18.69 \\
\hline My child's exercise has decreased as a result of COVID-19 & 135 & 67.50 \\
\hline My child's diet has been poorer as a result of COVID-19 & 22 & 11.22 \\
\hline My child's ability to spend time outside has reduced as a result of COVID. & 161 & 80.90 \\
\hline My child has difficulty following the recommendations for keeping away from close contact with people & 52 & 27.51 \\
\hline My child has had difficulty maintaining relationships as a result of COVID & 83 & 43.23 \\
\hline
\end{tabular}


Table 3 (Continued).

\begin{tabular}{|l|r|r|}
\hline & $\mathbf{n}$ & $\%$ \\
\hline My child has become easily annoyed, irritable, and angry since the COVID-I9 outbreak, compared to before the & 120 & $60.6 \mathrm{I}$ \\
outbreak & 52 \\
The cancellation of important events (such as holiday, birthday celebration etc.) has been difficult for my family & 27.96 \\
My child has been watching significantly more TV or digital media (eg, Netflix, YouTube, web surfing) since before & 144 \\
COVID & 73.10 \\
My child has been using significantly more social media (eg, Facetime, Facebook, Instagram, Snapchat, Twitter, TikTok) & 63 \\
since before COVID & 43.45 \\
My child has been playing significantly more video games since before COVID & 101 & 58.72 \\
\hline
\end{tabular}

Table 4 Correlations with Child and Parent Wellbeing

\begin{tabular}{|l|r|r|r|}
\hline & $\begin{array}{r}\text { Impact on Child's Overall } \\
\text { Wellbeing }\end{array}$ & $\begin{array}{r}\text { Worsening of Child Pre-Existing Health } \\
\text { Conditions }\end{array}$ & $\begin{array}{r}\text { Impact on Parental } \\
\text { Wellbeing }\end{array}$ \\
\hline Parent age & -0.066 & -0.131 & -0.111 \\
Child sex & -0.086 & 0.017 & 0.001 \\
Child age & 0.027 & -0.026 & 0.012 \\
Routines disrupted & $\mathbf{0 . 3 6 2}$ & $\mathbf{0 . 4 0 9}$ & $\mathbf{0 . 3 4 3}$ \\
Restrictions stressful & $\mathbf{0 . 4 2 6}$ & $\mathbf{0 . 4 6 9}$ & $\mathbf{0 . 3 0 5}$ \\
Home isolation stressful & $\mathbf{0 . 2 8 4}$ & $\mathbf{0 . 2 9 8}$ & 0.595 \\
Home learning has disrupted & 0.068 & 0.087 & \\
child's learning & & -0.062 & \\
Satisfaction with services child & -0.166 & & $-\mathbf{0 . 2 4 3}$ \\
has received & & & \\
\hline
\end{tabular}

Note: Significant correlations $(p<0.05)$ are shown in bold.

(27.96\%) had felt they were negatively impacted by the cancellation of holidays and family events. Increased negative emotions have been displayed by $60.61 \%$ of children. A full $73.10 \%$ of children were reportedly engaging more in consumption of screen-based media, with $43.45 \%$ reported to be using more social media, and $58.72 \%$ reporting an increase in use of video games.

A small majority of caregivers $(60.00 \%)$ felt the pandemic had changed the way they spent time with their child, with $71.86 \%$ reported having a good or very good relationship with their child. Only $12.99 \%$ felt their child's relationships with their siblings had worsened, and only $11.50 \%$ reported that there were unmet service needs for their child during this time.

Participants were asked to reflect on whether they saw any "good" come out of the pandemic, and $76.00 \%$ responded that they had. Notably, $71.50 \%$ reported spending more time with family, and $55.00 \%$ reported learning new skills such as cooking or gardening. However, most disagreed that they had been more strongly connected in other ways; $78.00 \%$ had not stayed in touch with extended family more often than usual, $90.00 \%$ had not seen friends more than usual, $99.00 \%$ had not formed new connections using online methods, $93.50 \%$ reported they had not returned to any form of religion or spirituality, and $91.50 \%$ had not engaged in home-based activities such as meditation or mindfulness.

A simple correlational analysis was conducted to explore areas associated with impact on children, worsening of children's pre-existing health conditions, and impact on parents (see Table 4). It was found that greater disruption of routines, higher stress in relation to restrictions, and higher stress in relation to home isolation were all related to greater impact on child wellbeing, greater impact on parent wellbeing, and more worsening of pre-existing health conditions for children (all $\mathrm{p}<0.001$ ). Parent age, child age, and child sex were not related to any of the impact variables. Interestingly, a sense of home learning having disrupted the child's learning was not significantly correlated with any of the wellbeing variables. A lower level of parental satisfaction with services received by the child was related to higher impact on parent wellbeing 
( $\mathrm{p}<0.01$ ), but not to child wellbeing or worsening of child's health conditions.

\section{Discussion}

As was expected based on previous research, ${ }^{11}$ large proportions of families reported a worsening of their children's symptoms. More than half reported overall negative health impacts for children, while large proportions within each diagnostic category also reported worsening symptoms. A little over $60 \%$ reported increased emotional lability since before the start of the pandemic. Add to this that a large proportion of parents felt their own wellbeing had been impacted and a large proportion felt their other children had experienced a significant impact from the pandemic, and it becomes clear that this particular population of families of a child with a neurodevelopmental disorder are at significant risk of deteriorating mental health as the COVID-19 emergency continues on.

Homeschooling was identified as a significant stress in the current study, as it has been in previously cited research. ${ }^{6}$ However, results indicate that in the current context this was generally not because parents felt incapable or resources were not available, but because less than half of respondents felt their child had sufficient capacity to engage in homebased learning. For families of children with neurodevelopmental disorders, it appears that the difference between in person and remote schooling is particularly wide, suggesting impacts on education for affected children might also be expected to be significant as they step into the future. Similarly, with around three out of four families not being able to access professional support as usual, treatment gains might be expected to lag for this cohort unless additional treatment support is made available. In spite of these things, however, the correlational analysis showed that homeschooling and disruption of services were largely not connected to child wellbeing, but that disruption of routine, restrictions, and home isolation were key correlates.

It is noteworthy that the current sample, consisting mostly of mothers, indicated markedly low wellbeing while indicating only a moderate level of mental health problems. It seems to suggest that impacts are occurring in smaller, subtler, and perhaps slower ways during the current crisis, rather than leading to a sense in parents that they cannot cope. Whilst this may be protective for families as they navigate the ongoing crisis, it remains to be seen what effects may occur for parents downstream and what health and wellbeing needs may have developed over a prolonged period of additional stress and danger.
This, too, may be an area requiring additional resources for families of children with neurodevelopmental and psychological disorders if they are to continue to maintain resilience moving forward.

The strengths of the current study include that it provides timely information on the impact of COVID-19 on children with neurodevelopmental disorders and their families while the pandemic is still ongoing. Respondents have provided information about many angles of the family - parents, children, siblings, mental health, physical health, economic health, education, treatment needs - allowing for a contextualized understanding of mental health needs against the backdrop of other aspects of everyday life. There are, however, a number of limitations of the study. The self-report nature of the questionnaire means there is no objective data about symptoms or diagnoses - only the report of the family as to whether presentations have improved or worsened - and that these self-reports were stated in telephone interview, which could conceivably have introduced some bias in responding.

Additionally, the parents/caregivers were asked to respond to questions about their child's wellbeing, rather than children and young people being able to be directly accessed to generate this information. This means potentially important information may not have been included here, relating to children or young people whose caregivers are not aware of the extent of their needs or experiences. Finally, no causality was able to be demonstrated with a pre- and post-COVID measure; it must be inferred by respondents' indications that situations have changed since the beginning of the pandemic.

In conclusion, current results highlight a growing area of need amongst families of children with neurodevelopmental or psychological disorders. A reduction in service availability and a reduction in children's ability to engage in education and schooling could be expected to lead to significant additional burdens of care well after the eventual end of the COVID-19 crisis if additional resources and supports are not strategically deployed to support children and families in need.

\section{Data Sharing Statement}

The authors will make de-identified data available upon request.

\section{Acknowledgments}

The authors would like to thank all those who responded to the survey and acknowledge the assistance of Valentina Laganà in data collection. 


\section{Funding}

This research received no specific grant from any funding agency in the public, commercial or not-for-profit sectors.

\section{Disclosure}

The authors report no conflicts of interest in this work.

\section{References}

1. Crawford P. Editorial perspective: cabin fever - the impact of lockdown on children and young people. Child Adolesc Ment Health. 2021;26(2):167-168. doi:10.1111/camh.12458

2. Graber KM, Byrne EM, Goodacre EJ, et al. A rapid review of the impact of quarantine and restricted environments on children's play and the role of play in children's health. Child Care Health Dev. 2021;47(2):143-153.

3. Magson NR, Freeman JYA, Rapee RM, et al. Risk and protective factors for prospective changes in adolescent mental health during the COVID-19 pandemic. $J$ Youth Adolesc. 2021;50(1):44-57. doi:10.1007/s10964-020-01332-9

4. Lee SJ, Ward KP, Chang OD, Downing KM. Parenting activities and the transition to home-based education during the COVID-19 pandemic. Child Youth Serv Rev. 2021;122:105585. doi:10.1016/j. childyouth.2020.105585

5. Cost KT, Crosbie J, Anagnostou E, et al. Mostly worse, occasionally better: impact of covid-19 pandemic on the mental health of Canadian children and adolescents. Eur Child Adolesc Psychiatry. 2021:1-14. doi:10.1007/s00787-021-01744-3

6. Thorell LB, Skoglund C, de la Peña AG, et al. Parental experiences of homeschooling during the covid-19 pandemic: differences between seven European countries and between children with and without mental health conditions. Eur Child Adolesc Psychiatry. 2021:1-13. doi:10.1007/s00787-020-01706-1
7. Fong V C, Iarocci G. Child and family outcomes following pandemics: a systematic review and recommendations on COVID-19 policies. J Pediatr Psychol. 2020;45(10):1124-1143. doi:10.1093/ jpepsy/jsaa092

8. Amendola S, Spensieri V, Hengartner MP, Cerutti R. Mental health of Italian adults during covid-19 pandemic. $\mathrm{Br} J$ Health Psychol. 2021;26(2):644-656. doi:10.1111/bjhp. 12502

9. Paulauskaite L, Farris O, Spencer H, Absoud A. My son can't socially distance or wear a mask: how families of preschool children with severe developmental delays and challenging behavior experienced the covid-19 pandemic. J Ment Health Res Intellect Disabil. 2021;14(2):225-236. doi:10.1080/19315864.2021.1874578

10. Grumi S, Provenzi L, Gardani A, et al. Rehabilitation services lockdown during the COVID-19 emergency: the mental health response of caregivers of children with neurodevelopmental disabilities. Disabil Rehabil. 2021;43(1):27-32. doi:10.1080/09638288.2020.1842520

11. Masi A, Mendoza Diaz A, Tully L, et al. Impact of the COVID-19 pandemic on the well-being of children with neurodevelopmental disabilities and their parents. J Paediatr Child Health. 2021;57 (5):631-636. doi:10.1111/jpc.15285

12. Kessler RC, Barker PR, Colpe LJ, et al. Screening for serious mental illness in the general population. Arch Gen Psychiatry. 2003;60 (2):184-189. doi:10.1001/archpsyc.60.2.184

13. Kroenke K, Spitzer RL, Williams JB, Monahan PO, Lowe B. Anxiety disorders in primary care: prevalence, impairment, comorbidity, and detection. Ann Intern Med. 2007;146:317-325. doi:10.7326/00034819-146-5-200703060-00004

14. World Health Organization. Wellbeing Measures in Primary Health Care/ the Depcare Project. Copenhagen: WHO Regional Office for Europe; 1998.
Neuropsychiatric Disease and Treatment

\section{Publish your work in this journal}

Neuropsychiatric Disease and Treatment is an international, peerreviewed journal of clinical therapeutics and pharmacology focusing on concise rapid reporting of clinical or pre-clinical studies on a range of neuropsychiatric and neurological disorders. This journal is indexed on PubMed Central, the 'PsycINFO' database and CAS, and

\section{Dovepress}

is the official journal of The International Neuropsychiatric Association (INA). The manuscript management system is completely online and includes a very quick and fair peer-review system, which is all easy to use. Visit http://www.dovepress.com/testimonials.php to read real quotes from published authors. 